\title{
Lidocaine exerts anticancer activity in bladder cancer by targeting isoprenylcysteine carboxylmethyltransferase (ICMT)
}

\author{
Xiaodan Teng, Yang Liu, Liping Wang, Guonian Wang \\ Department of Anesthesiology, Harbin Medical University Cancer Hospital, Harbin, China \\ Contributions: (I) Conception and design: X Teng, Y Liu; (II) Administrative support: G Wang; (III) Provision of study materials or patients: Y Liu, \\ L Wang; (IV) Collection and assembly of data: X Teng, L Wang; (V) Data analysis and interpretation: X Teng, Y Liu, G Wang; (VI) Manuscript \\ writing: All authors; (VII) Final approval of manuscript: All authors. \\ Correspondence to: Guonian Wang. Harbin Medical University Cancer Hospital, No. 150 Haping Street, Nangang District, Harbin 150086, China. \\ Email: t345254900@163.com.
}

\begin{abstract}
Background: Bladder cancer is one of the most common malignant tumors among humans and has a high mortality. Clinically, lidocaine is the most commonly used local anesthetic, which can inhibit the proliferation of bladder cancer cells; however, its downstream specific molecular mechanisms are unclear.

Methods: The Swiss Target and TargetNet databases were used to analyze the target of lidocaine. The online public cancer transcriptome database UALCAN was used to analyze the up-regulated genes in The Cancer Genome Atlas Urothelial Bladder Carcinoma (TCGA-BLCA) data collection, and the intersection of the 2 was used to obtain the core target. The only target, isoprenylcysteine carboxylmethyltransferase (ICMT), was obtained by combining the correlation between the target and the clinical information of bladder cancer and the Kaplan-Meier (K-M) survival curve. Then, UMUC3 and T24 cells were selected as research vectors in vitro. Cell proliferation, cell cycle, and apoptosis were detected by cell counting kit-8, colony formation, flow cytometry, and western blotting.

Results: Network pharmacology analysis showed that ICMT might be one of the targets of lidocaine, and the expression level of ICMT was closely related to the clinical phenotype of bladder cancer. Lidocaine treatment (4 and $8 \mathrm{mM}$ ) significantly inhibited the proliferation of UMUC3 and T24 cells, promoted apoptosis, and significantly inhibited the mass and volume of xenograft tumors. In vitro experiments showed that ICMT promoted the proliferation of UMUC3 and T24 cells. Lidocaine inhibited the expression of ICMT in UMUC3 and T24 cells in a concentration and time-dependent manner, and inhibited cell proliferation by down-regulating ICMT expression.
\end{abstract}

Conclusions: Lidocaine exerts anti-tumor effect by down-regulating the expression of ICMT in bladder cancer.

Keywords: Bladder cancer; lidocaine; isoprenylcysteine carboxylmethyltransferase (ICMT); proliferation; apoptosis

Submitted Sep 09, 2021. Accepted for publication Nov 03, 2021.

doi: $10.21037 /$ tau-21-893

View this article at: https://dx.doi.org/10.21037/tau-21-893

\section{Introduction}

Bladder cancer is one of the most common malignant tumors of the urinary system and the 11th most frequently diagnosed cancer in the world (1). Although significant advances have been made in surgical techniques and adjuvant therapy, the prognosis of bladder cancer remains poor. Therefore, understanding the molecular mechanism of bladder cancer is helpful to the exploration of effective diagnostic and prognostic markers of bladder cancer.

Lidocaine is a local anesthetic, which is widely clinically used in local anesthesia and analgesia. Recently, it has been found that subcutaneous infusion of lidocaine can be safely 
used for cancer pain management (2). In addition, lidocaine also has good anti-tumor therapeutic potential. Lidocaine reduces the survival rate and colony formation ability of HepG2 cells by up-regulating the expression of CPEB3 (3). In human thyroid carcinoma cells, lidocaine induces apoptosis by regulating mitogen activated protein kinase (MAPK) pathway (4). Lidocaine can inhibit the proliferation of bladder cancer cells alone and enhance the anticancer effect of other chemotherapeutic drugs (5). However, the downstream molecular mechanism of lidocaine in regulating the proliferation of bladder cancer cells is unclear.

The final step of isopreanoidization of translated proteins catalyzed by isoprenylcysteine carboxylmethyltransferase (ICMT) is crucial to the stability and normal function of many carcinogenic proteins. Recent studies have shown that the expression of ICMT is up-regulated in ovarian cancer and cervical cancer, and that it promotes a variety of malignant biological behaviors of tumor cells, such as growth, migration, and survival (6-8). Consistently, ICMT inhibition has exhibited anticancer activity in cancers including pancreatic cancer, leukemia, and cervical cancer (9-11).

In this study, we obtained ICMT as one of the targets of lidocaine in bladder cancer patients through network pharmacology analysis. The expression of ICMT in tissue samples of bladder cancer patients analyzed from The Cancer Genome Atlas (TCGA) database was significantly increased, and the high expression of ICMT was related to poor tumor grade, poor pathological $\mathrm{T}$ stage, and high recurrence rate. In vitro, ICMT promotes the proliferation of bladder cancer cells, whereas lidocaine inhibits cell survival and growth by downregulating ICMT. In this study, we investigated the mechanism of lidocaine on bladder cancer cell proliferation. This study has laid the foundation for lidocaine treatment of bladder cancer. We present the following article in accordance with the ARRIVE reporting checklist (available at https://dx.doi. org/10.21037/tau-21-893).

\section{Methods}

\section{Computational target fishing using TargetNet and SwissTarget}

The SDF file of lidocaine (Pubchem CID: 3676) was downloaded from the Pubchem database and entered into TargetNet (https://targetnet.scbdd.com/) and SwissTarget (https://www.swisstargetprediction.ch/) servers. The default values were set for all parameter settings, and the protein targets identified by the 2 servers were allowed to overlap. Therefore, they can be considered as preselected targets for further research.

\section{UALCAN database}

The online public cancer transcriptome database UALCAN (ualcan.path.uab.edu/) was used to analyze the upregulated genes in The Cancer Genome Atlas Urothelial Bladder Carcinoma (TCGA-BLCA). According to the clinicopathological parameters, such as tumor $\mathrm{T}$ stage and tumor grade, the gene expression profiles in bladder cancer samples were analyzed, and the target genes were screened. Subsequently, Kaplan-Meier (K-M) survival analysis was performed on the target genes to explore the value and significance of a single gene in the prognosis prediction of bladder cancer. The study was conducted in accordance with the Declaration of Helsinki (as revised in 2013).

\section{Cell culture and transfection}

Human bladder cancer cell lines (UMUC3 and T24) were purchased from American Type Culture Collection (ATCC; Manassas, VA, USA). Cells were cultured at $37{ }^{\circ} \mathrm{C}$ in $5 \% \mathrm{CO}_{2}$ in Roswell Park Memorial Institute (RPMI)-1640 culture medium (Gibco, Gaithersburg, MD, USA) containing $10 \%$ fetal bovine serum (FBS; Gibco), and subcultured at 80-90\% cell density. According to the standard procedure, ICMT overexpression plasmid (Addgene, Watertown, MA, USA; Plasmid \# 29272) was transfected into cells by Lipofectamine 2000 (Invitrogen, Carlsbad, CA, USA). Then, ICMT short hairpin RNA (shRNA) expressing plasmids were constructed in lentiviral vector PLL3.7. by (GenePharma, Shanghai, China). The ICMT target sequence was: (I) 5' -CCCTGTCATTGTTCCACTATT-3', (II) 5'-CTTGGTTTCGGCATCCTTCTT-3'. Cells were harvested for ICMT protein expression level analysis at $72 \mathrm{~h}$ post transfection.

\section{Cell proliferation test}

Cell counting Kit-8 (CCK-8; Beyotime, Shanghai, China) was used to explore cell proliferation. About $1 \times 10^{4}$ cells or transfected cells were seeded into triplicate wells of 96-well plates with $100 \mu \mathrm{L}$ medium per well. After $24 \mathrm{~h}$ of culture, cells were treated with different doses of lidocaine (Sigma- 
Aldrich, St. Louis, MO, USA) at different time points. The cells were then incubated with $10 \mu \mathrm{L}$ CCK-8 solution for $2 \mathrm{~h}$, and the absorbance was measured at $450 \mathrm{~nm}$.

\section{Colony formation assay}

After $48 \mathrm{~h}$ of culture with untreated, treated with lidocaine, or transfected cells, the cells were seeded into each well of the 6-well culture plate at a density of 500 cells/well. After 14 days of culture in drug-free culture, the cells were fixed with paraformaldehyde for $15 \mathrm{~min}$, and stained with crystal violet for $10 \mathrm{~min}$ to observe the colonies. Only $\geq 50$ cells were counted and compared as positive colonies.

\section{Flow cytometry detection of cell cycle distribution}

For cell cycle analysis, the specified treated UMUC3 and T24 cells were harvested and fixed overnight in $70 \%$ ethanol at $4{ }^{\circ} \mathrm{C}$. The cell lines were treated with $0.5 \mathrm{mg} / \mathrm{mL}$ RNase A (Keygen Biotech, Nanjing, China) and stained with $1 \mathrm{mg} / \mathrm{mL}$ propidium iodide (PI; Becton, Dickinson and Co. Biosciences (BD), San Diego, CA, USA) for $0.5 \mathrm{~h}$ at $37^{\circ} \mathrm{C}$. We then used FACSCalibur flow cytometry (BD, San Jose, CA, USA) was used to determine the distribution of cell cycle stages. The percentages of G0/G1, S, and G2/M cells were calculated.

\section{Flow cytometry detection of apoptosis}

For apoptosis analysis, UMUC3 and T24 cells were harvested and washed twice with cold phosphate-buffered saline (PBS) and suspended in binding buffer containing V-FITC and PI for $15 \mathrm{~min}$ at room temperature. The FACSCalibur flow cytometry was used to analyze apoptosis.

\section{Western blot analysis}

The cells to be tested were lysed in radioimmunoprecipitation assay (RIPA) buffer (Beyotime) and centrifuged at $12,000 \mathrm{rpm}$ for $15 \mathrm{~min}$ at $4{ }^{\circ} \mathrm{C}$. The supernatant was collected and the total protein content was determined by bicinchoninic acid (BCA) Protein Assay Kit (Beyotime). The same protein $(40 \mu \mathrm{g} / \mathrm{lane})$ was separated on sodium dodecyl sulfate-polyacrylamide gel electrophoresis (SDSPAGE) gel and transferred to polyvinylidene fluoride (PVDF) membrane (Millipore, Danvers, MA, USA). The membrane was sealed with $5 \%$ skimmed milk and incubated with primary antibodies against Ki67 (1:3,000; ab92742,
Abcam, Cambridge, MA, USA), CDK2 (1:1,000; ab32147, Abcam), CCNB1 (1:10,000; ab32053, Abcam), BAX (1:1,000; ab32503, Abcam), BCL2 (1:500; ab32124, Abcam), and $\beta$-actin (1:2,000; ab6276, Abcam) overnight at $4{ }^{\circ} \mathrm{C}$. The next day, after washing with PBS/Tween (PBST) for $3 \times 5 \mathrm{~min}$, the membrane was incubated with secondary antibody at $37{ }^{\circ} \mathrm{C}$ for $2 \mathrm{~h}$. After washing, Tanon-5200 image analyzer (Tanon Science and Technology, Shanghai, China) was used to quantify protein bands.

\section{Lactate dehydrogenase (LDH) cytotoxicity test}

LDH assay was performed using D-Plus TM LDH cytotoxicity assay kit (Dongin Biotech, Seoul, Korea). In short, $2.5 \times 10^{4}$ cells per well were seeded in 96-well plates. After incubation for $24 \mathrm{~h}$, the specified dose of lidocaine was added to each hole, and the final volume was $100 \mu \mathrm{L}$. After $48 \mathrm{~h}$, the solution was centrifuged at $600 \times \mathrm{g}$ for $10 \mathrm{~min}$ to remove floating cells from the supernatant. The control cells were lysed by adding $10 \mu \mathrm{L}$ lysis buffer before centrifugation. Each supernatant $(10 \mu \mathrm{L})$ was transferred to a new hole in the 96-well plate. Finally, $100 \mu \mathrm{L} \mathrm{LDH}$ reaction mixture (the ratio of WST substrate to $\mathrm{LDH}$ determination buffer was 1:50) was added, and the samples were incubated at $25{ }^{\circ} \mathrm{C}$ for $30 \mathrm{~min}$. The absorbance at $450 \mathrm{~nm}$ was measured by a microplate reader. We conducted 3 independent experiments in duplicate at different time points.

\section{Nude mice xenograft model}

A total of 16 male BALB/c-nu/nu mice, aged 4-6 weeks and weighing 18-22 g, were purchased from Vital River Laboratories (Beijing, China). They were raised under specific pathogen free (SPF) conditions with a 12-h light/ dark cycle in a temperature-controlled facility $\left(22^{\circ} \mathrm{C}\right)$ with access to chow and water ad libitum. Mice were randomly divided into 2 groups: control group and lidocaine $(8$ mice in each group). Mice in the lidocaine group were injected with $5 \times 10^{6} \mathrm{~T} 24$ cells into the right abdominal cavity, and lidocaine $(100 \mathrm{mg} / \mathrm{kg})$ was intravenously injected 3 times every week (12). The mice in the control group were injected with T24 cells and an equal volume of normal saline 3 times in the right abdominal cavity. After 14 days, the mice were sacrificed by cervical dislocation. Calculation of tumor volume and weight was performed as described above (13). Experiments were performed under a project license (No. 2020067) granted by ethics committee of 
A
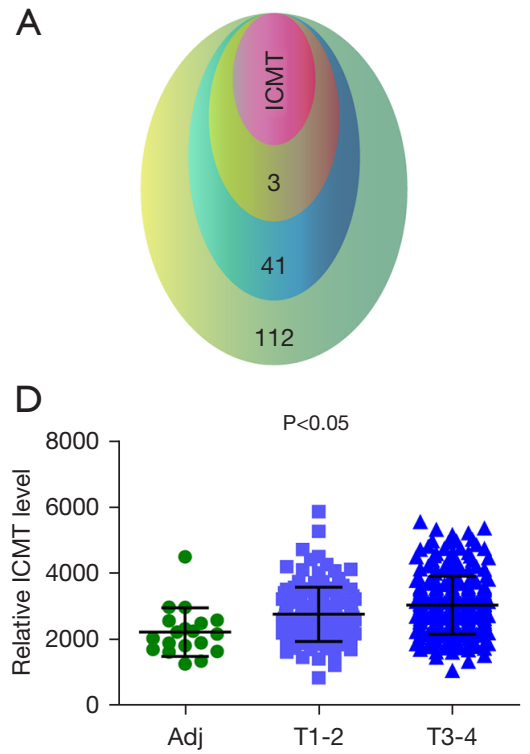

G

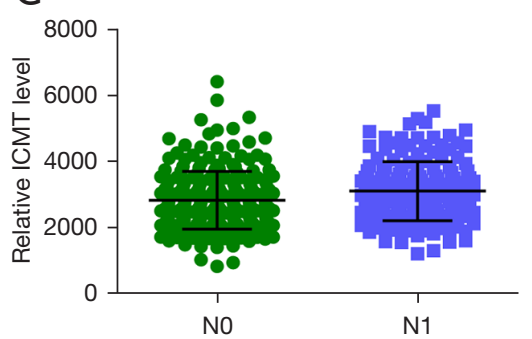

B

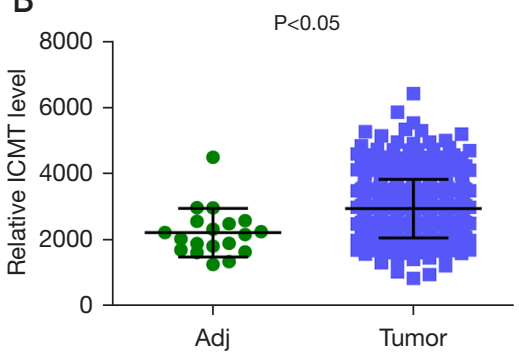

E

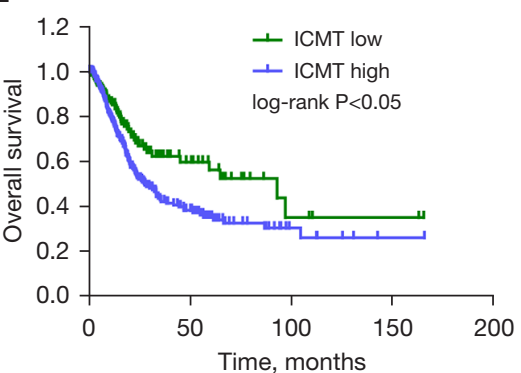

$\mathrm{H}$

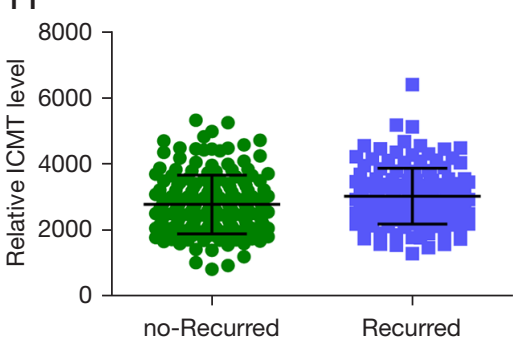

C

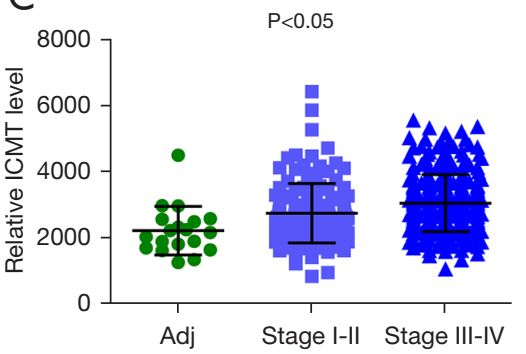

$\mathrm{F}$

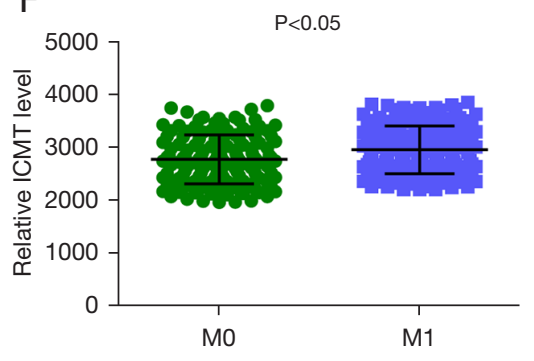

Figure 1 Network pharmacology analysis of lidocaine targets in bladder cancer. (A) The prediction schematic diagram of the core target of lidocaine; (B) the expression of ICMT in TCGA-BLCA; (C) the expression of ICMT and the tumor grade of BLCA; (D) the expression of ICMT and the T stage of BLCA tumor; (E) K-M curve was used to analyze the relationship between the expression of ICMT and the survival of BLCA. The expression level of ICMT was correlated with distal metastasis $(\mathrm{F})$, lymph node metastasis $(\mathrm{G})$ and recurrence $(\mathrm{H})$ of bladder cancer. $\mathrm{P}<0.05$ was considered statistically significant. TCGA-BLCA, The Cancer Genome Atlas Urothelial Bladder Carcinoma; K-M, Kaplan-Meier.

Harbin Medical University Cancer Hospital, in compliance with Animal Management Rule of China (Ministry of Health, China, document No. 552001). A protocol was prepared before the study without registration.

\section{Statistical analysis}

Data were expressed as mean \pm SD for at least 3 independent experiments. The software SPSS 19.0 (IBM Corp., Armonk, NY, USA) was used for statistical analysis. The statistical evaluation of the data was carried out by using unpaired Student's $t$-test and analysis of variance (ANOVA) by a post-hoc test. A $\mathrm{P}$ value $<0.05$ was considered $\mathrm{t}$ statistically significant and graphs were created with GraphPad Prism 6.0 software (GraphPad Software, San Diego, CA, USA).

\section{Results}

\section{Network pharmacology predicts the target of lidocaine and the clinical significance of ICMT in bladder cancer patients}

We used Swiss Target (pro $>0$ ) and TargetNet (pro $>0$ ) to predict the target of lidocaine. The 2 were combined to obtain 157 targets of lidocaine (Figure 1A). The UALCAN online database was used to analyze the up-regulated genes in TCGA-BLCA, and the results showed that 45 genes, including ICMT, were up-regulated (Figure 1B). 
The TCGA-BLCA data matrix and clinical information were downloaded to analyze the relationship between the expression levels of these 44 genes and the tumor grade and T staging of BLCA. The results showed that only ICMT, DHODH, DRD4, and FAP increased with the progression of tumor grade and $\mathrm{T}$ staging. The expression trend of ICMT is shown in Figure 1C,1D. In addition, we further analyzed the relationship between these 4 genes and BLCA survival. The K-M curve showed that only ICMT was associated with BCLA survival (Figure 1E). Therefore, this study selected $I C M T$ as the research object. Subsequently, we found that the higher the expression of ICMT, the higher the incidence of distant metastasis, lymph node metastasis, and recurrence rate of bladder cancer (Figure $1 F-1 H$ ).

\section{Inbibition of proliferation and cycle of bladder cancer cells by lidocaine in vitro}

The chemosensitivity of UMUC3 and T24 cells to lidocaine was determined by CCK- 8 assay. The results showed that lidocaine inhibited the survival of UMUC3 and T24 cells, and the inhibition rate increased with the increase of drug treatment time and concentration. The UMUC3 and T24 cells were treated with 4 and $8 \mathrm{mM}$ lidocaine for $48 \mathrm{~h}$, respectively, and the cell inhibition rate reached about $50 \%$ (Figure 2A,2B). Therefore, UMUC3 and T24 cells were treated with lidocaine at the concentrations of 4 and $8 \mathrm{mM}$ for $48 \mathrm{~h}$, respectively. As shown in Figure 2C,2D, compared with the control group, lidocaine significantly reduced the optical density (OD) value. In addition, we evaluated the colony formation ability of the treated cells. As shown in Figure 2E, lidocaine at 4 and $8 \mathrm{mM}$ for $48 \mathrm{~h}$ significantly reduced the colony formation ability of UMUC3 and T24 cells, indicating that lidocaine inhibited the proliferation of bladder cancer cells. Next, in order to investigate whether lidocaine mediates the inhibitory effect of bladder cancer cell growth through cell cycle arrest mechanism, we used flow cytometry (PI staining) to analyze the cell cycle distribution after $48 \mathrm{~h}$ of treatment with the specified concentration of lidocaine. As shown in Figure 2F,2G, compared with the control group, lidocaine treatment significantly increased the proportion of cells in G0/G1 phase and decreased the proportion of cells in S phase, suggesting that lidocaine inhibited the growth of bladder cancer cells, partly due to the induction of cell cycle arrest in G1/S phase. Secondly, lidocaine significantly inhibited the protein expression of Ki67, CDK2, and CCNB1 in UMUC3 and T24 cells (Figure $2 H$ ).

\section{Lidocaine promotes apoptosis of bladder cancer cells in vitro}

The LDH cytotoxicity test is a colorimetric method, which provides a simple and reliable method for the determination of cytotoxicity. In UMUC3 and T24 cells, the LDH cytotoxicity of cells treated with the specified concentration of lidocaine was significantly higher than that of the control group (Figure $3 A$ ). Then, flow cytometry was used to detect the apoptosis of UMUC3 and T24 cells after lidocaine treatment. Lidocaine significantly increased the proportion of apoptotic cells in UMUC3 and T24 cells (Figure 3B). In order to further study the mechanism of this phenomenon, the expression level of apoptosis-related proteins was measured by western blotting. After lidocaine treatment, BAX levels in UMUC3 and T24 cells increased, while BCL2 levels decreased (Figure 3C), indicating that lidocaine may induce apoptosis by activating endogenous apoptotic pathways.

\section{Lidocaine inhibits bladder cancer cell proliferation in vivo}

To evaluate the antitumor effect of lidocaine in vivo, a T24 cell subcutaneous transplantation model was established. The body weight and tumor volume of mice were positively correlated with tumor severity. As shown in Figure 4, the tumor volume (Figure 4B,4C) and tumor weight (Figure $4 D$ ) of the control animals transplanted with T24 cancer cells were increased. Compared with the control group, the tumor volume (Figure $4 B, 4 C$ ) and tumor weight (Figure $4 D$ ) in the lidocaine group were significantly decreased.

\section{ICMT promotes bladder cancer cell proliferation in vitro}

In order to understand the effect of ICMT on the proliferation of bladder cancer cells, we used the methods of functional loss and functional acquisition to detect the proliferation. As shown in Figure 5, we found that ICMT overexpression significantly increased the OD values of UMUC3 and T24 cells (Figure 5B,5C), promoted the cell colony formation ability (Figure $5 D$ ), and up-regulated the Ki67 protein level (Figure 5E), suggesting that overexpression of ICMT gene significantly promoted cell survival and proliferation. Contrary to the promotion of overexpression of ICMT, knockdown of ICMT by shRNA significantly decreased the OD value of UMUC3 and T24 cells (Figure 5G,5H), inhibited the colony formation ability 
A

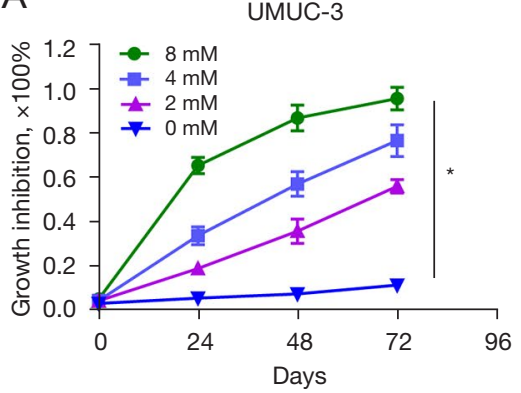

D

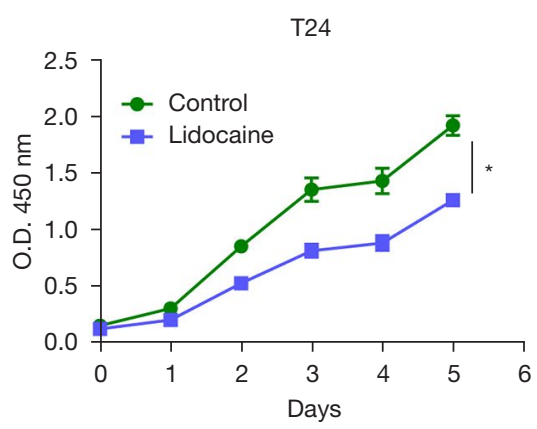

$\mathrm{F}$

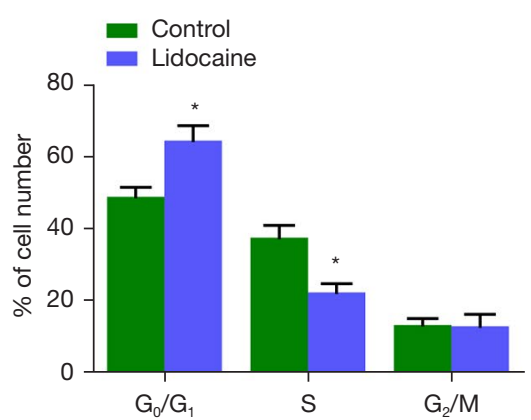

B

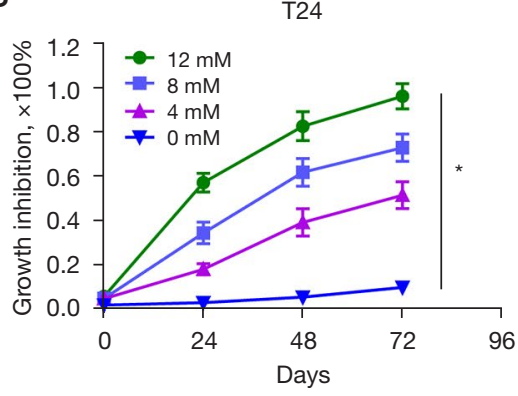

$E$

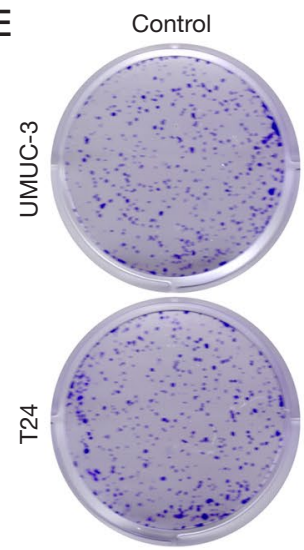

G

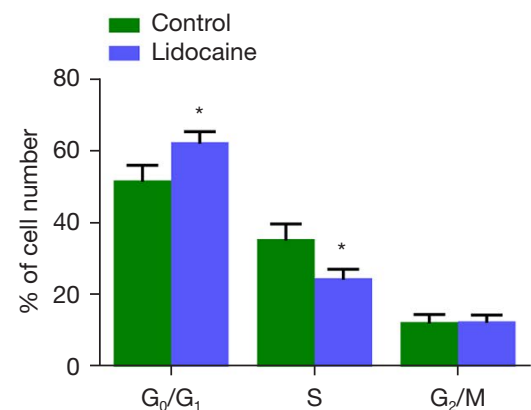

C

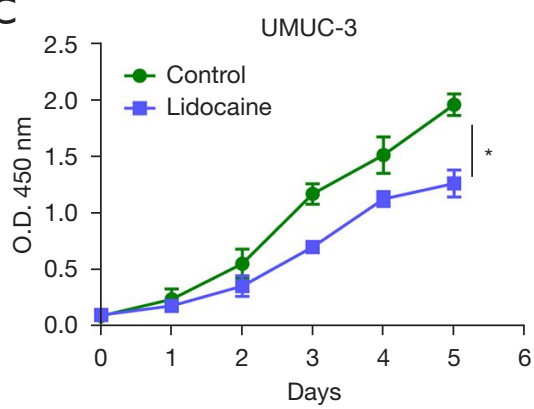

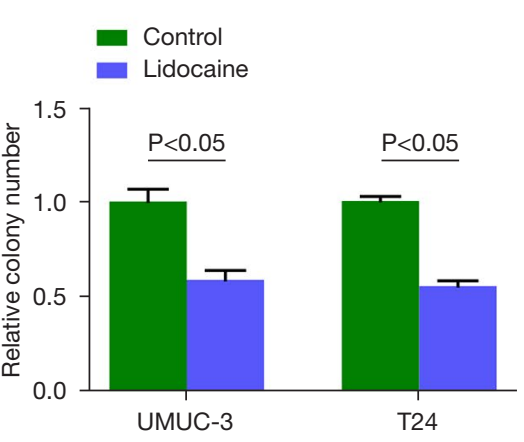

$\mathrm{H}$

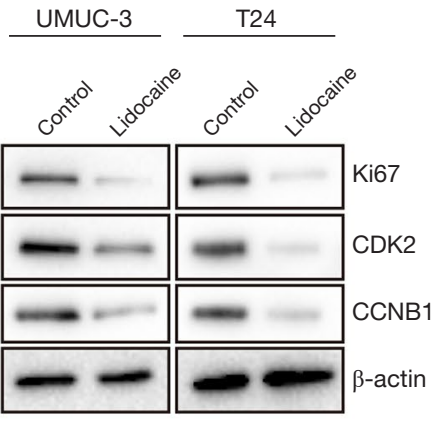

Figure 2 Effects of lidocaine on the proliferation and cycle of bladder cancer cells in vitro. (A,B) CCK-8 was used to detect the survival rates of UMUC3 and T24 cells after 24, 48, and $72 \mathrm{~h}$ treatment with different concentrations of lidocaine; (C,D) CCK-8 was used to detect the OD values of UMUC3 and T24 cells treated with 4 and $8 \mathrm{mM}$ lidocaine at different time points; (E) a colony formation assay (crystal violet staining) was performed to determine the clonogenic capacity of UMUC3 and T24 cells; (F,G) quantification of G0/G1, S, and G2/M phase cells in lidocaine-treated UMUC3 and T24 cells at the indicated concentrations (PI staining); (H) expression levels of ki67, CDK2, and CCNB1 were examined by western blotting in UMUC3 and T24 cells treated with lidocaine for $48 \mathrm{~h}$ at different concentrations. $\beta$-actin was used as the loading control. Data are presented as the mean $\pm \mathrm{SD}$. *, $\mathrm{P}<0.05$ vs. control. Results are representative of at least 3 independent experiments. CCK-8, cell counting kit-8; OD, optical density; PI, propidium iodide.

of cells (Figure 5I), and down-regulated the protein level of Ki67 (Figure 57). In summary, these results clearly indicate that ICMT promotes the proliferation of bladder cancer cells.

\section{Lidocaine inhibits bladder cancer cell proliferation through ICMT}

Next, in order to further reveal the potential mechanism of lidocaine affecting UMUC3 and T24 cells, the protein 

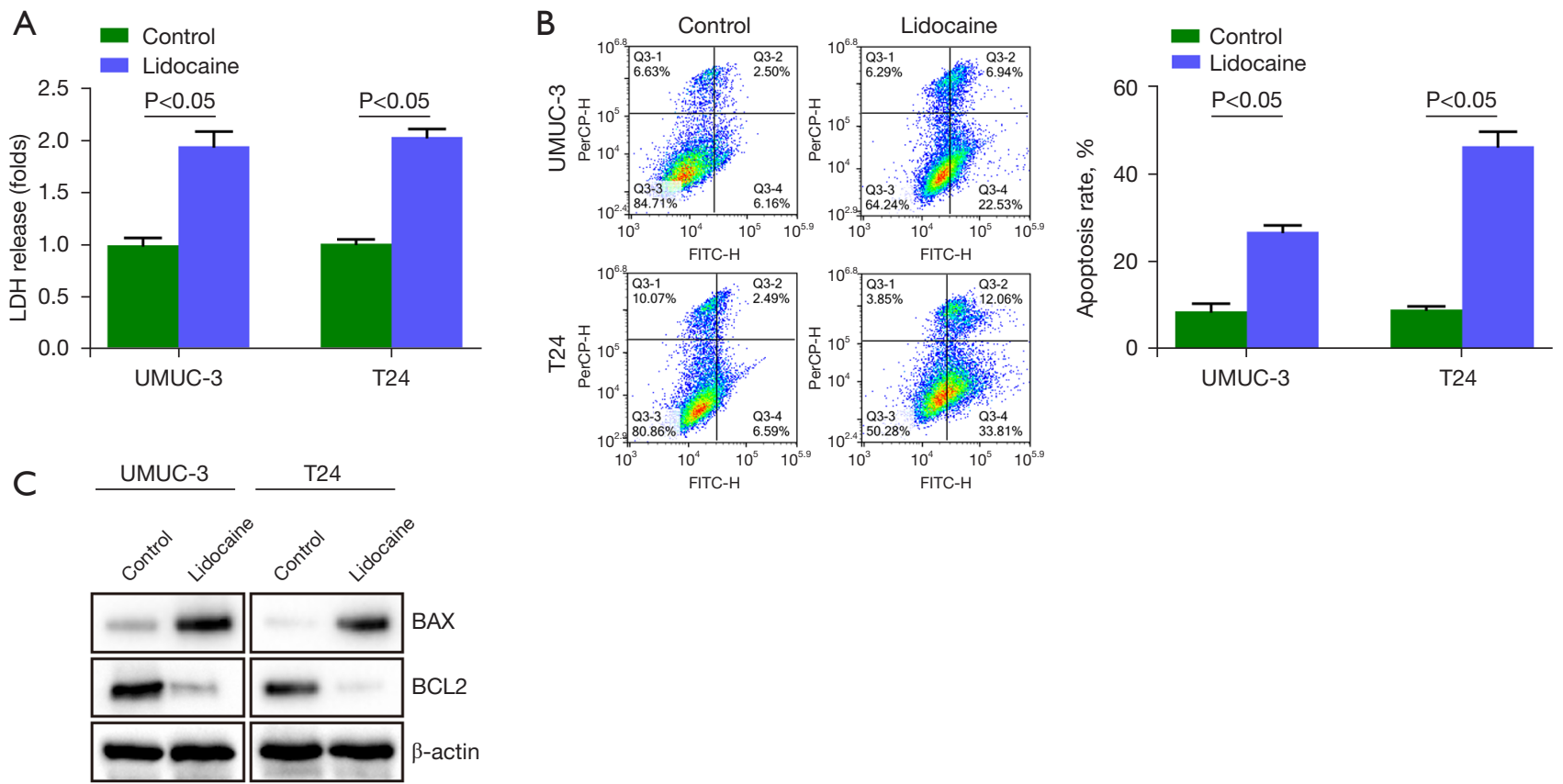

Figure 3 Effect of lidocaine on apoptosis of bladder cancer cells in vitro. (A) The cytotoxicity of lidocaine was evaluated by measuring the release of LDH. (B) The apoptosis induced by lidocaine was detected by annexin v-FITC and PI double staining. (C) Western blot was used to detect the expression levels of BAX and BCL2. $\beta$-actin was used as the loading control. Data are presented as the mean $\pm \mathrm{SD}$. * $\mathrm{P}<0.05 v$. control. Results are representative of at least 3 independent experiments. LDH, lactate dehydrogenase; PI, propidium iodide.
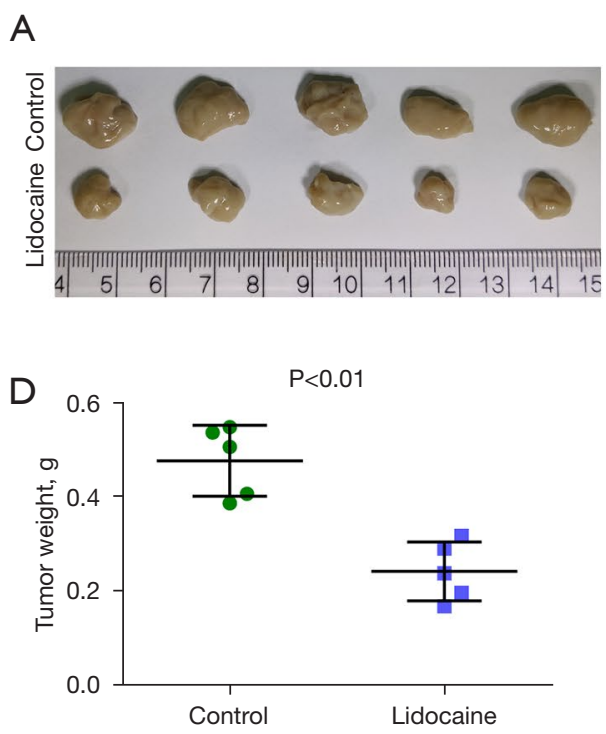
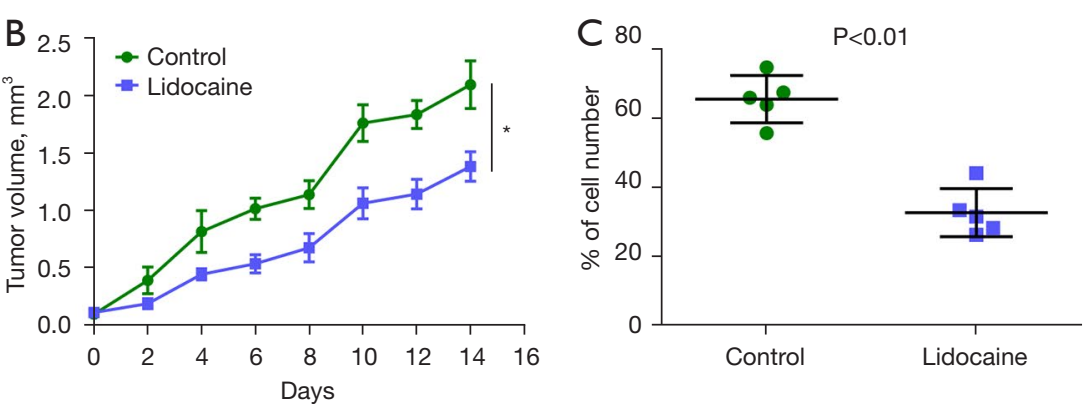

Figure 4 Antitumor effect of lidocaine on T24 tumor bearing nude mice. T24 cells were injected into right abdominal cavity of 4-6-week-old male nude mice. After injection of T24 cells, the nude mice were treated with normal saline and lidocaine (100 mg/kg) for 14 days, 3 times a week. (A) Tumor schematic diagram of nude mice 14 days after administration; (B) changes of tumor volume in nude mice within 14 days; (C) tumor volume on day 14; (D) tumor weight on day 14 . Data are presented as the mean $\pm \mathrm{SD}, \mathrm{n}=5$. *, $\mathrm{P}<0.05$ vs. control. 
A
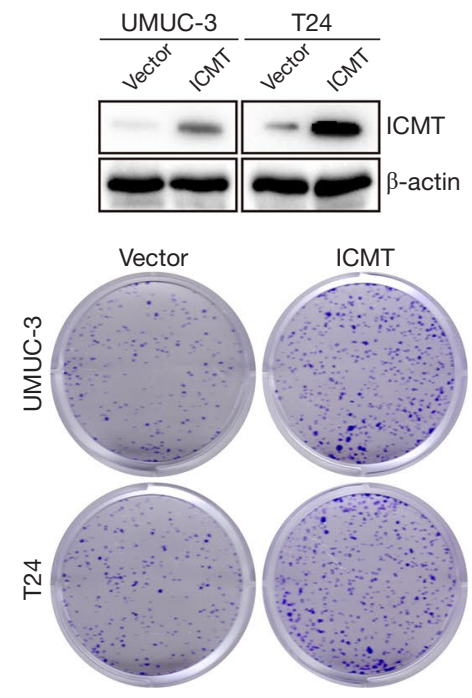

$\mathrm{F}$
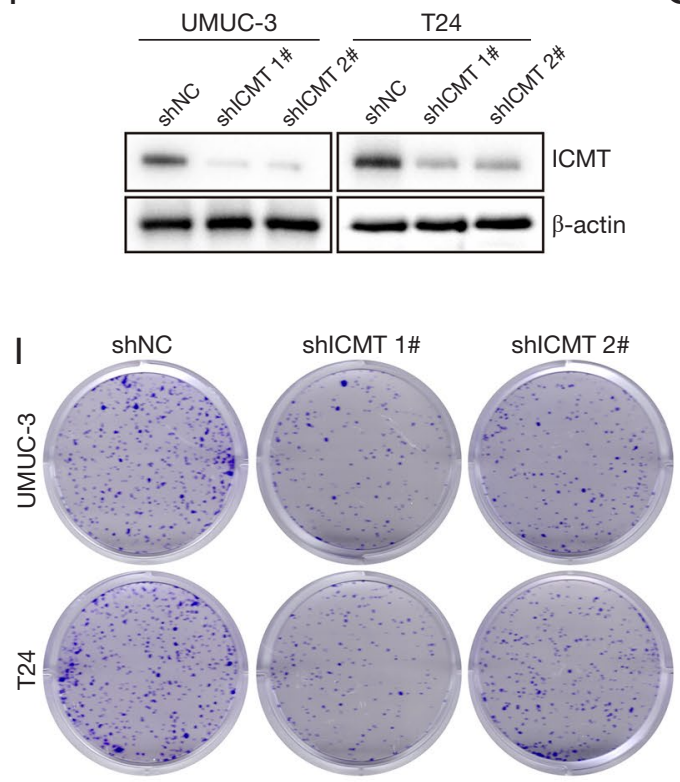

B
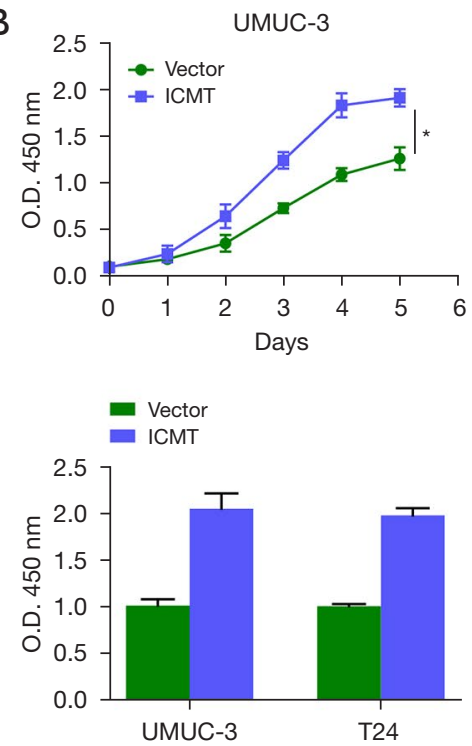

G
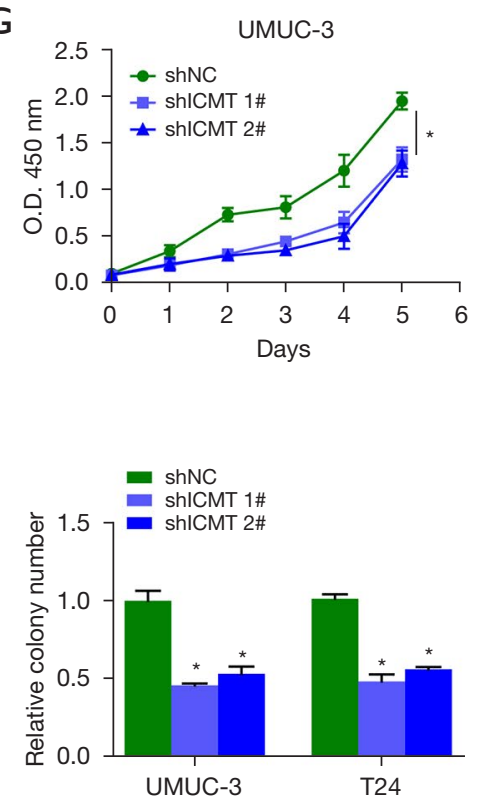

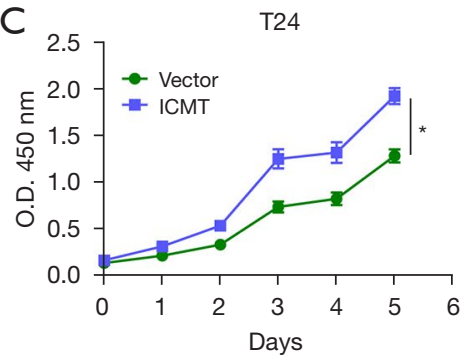

$E$
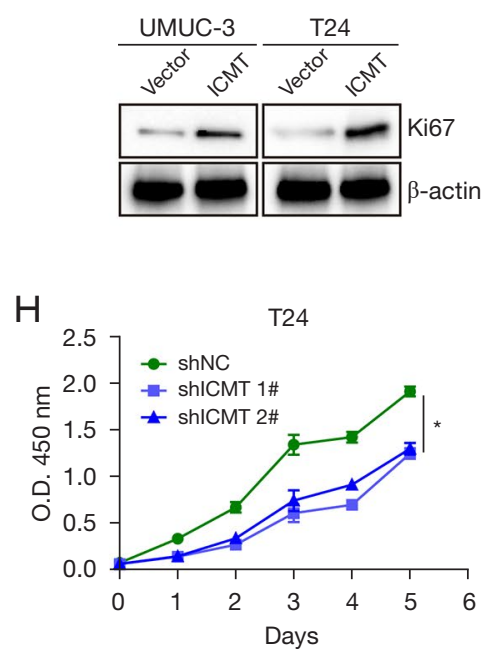

J

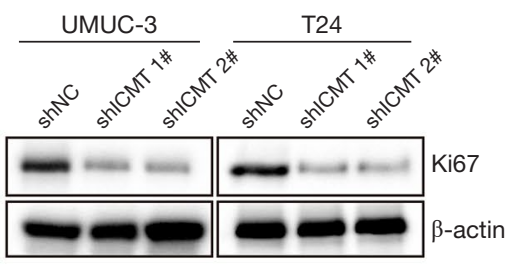

Figure 5 Effect of ICMT expression on the proliferation of bladder cancer cells. UMUC3 and T24 cells were transfected with ICMT overexpression or knockdown plasmids. Western blotting was used to analyze the protein expression levels of ICMT (A,F) and Ki67 (E,J). CCK-8 was used to detect the OD values of UMUC3 $(\mathrm{B}, \mathrm{G})$ and T24 cells $(\mathrm{C}, \mathrm{H})$. Colony formation assay (crystal violet staining) was used to determine the colony formation ability (D,I) of UMUC3 and T24 cells. Data are presented as the mean $\pm \mathrm{SD}$. *, $\mathrm{P}<0.05$ vs. control. Results are representative of at least 3 independent experiments. CCK-8, cell counting kit-8; OD, optical density.

expression of ICMT was detected by western blot analysis. As shown in Figure 6A, in response to lidocaine treatment, the expression levels of ICMT in UMUC3 and T24 cells decreased in a concentration-dependent manner. Then, the expression changes of ICMT in UMUC3 and T24 cells treated with 4 and $8 \mathrm{mM}$ lidocaine for 12,24 , and $48 \mathrm{~h}$ were detected. It was observed that lidocaine down-regulated the expression level of ICMT in a time-dependent manner (Figure $6 B$ ), suggesting that the expression of ICMT was regulated by lidocaine. We further treated cells with 

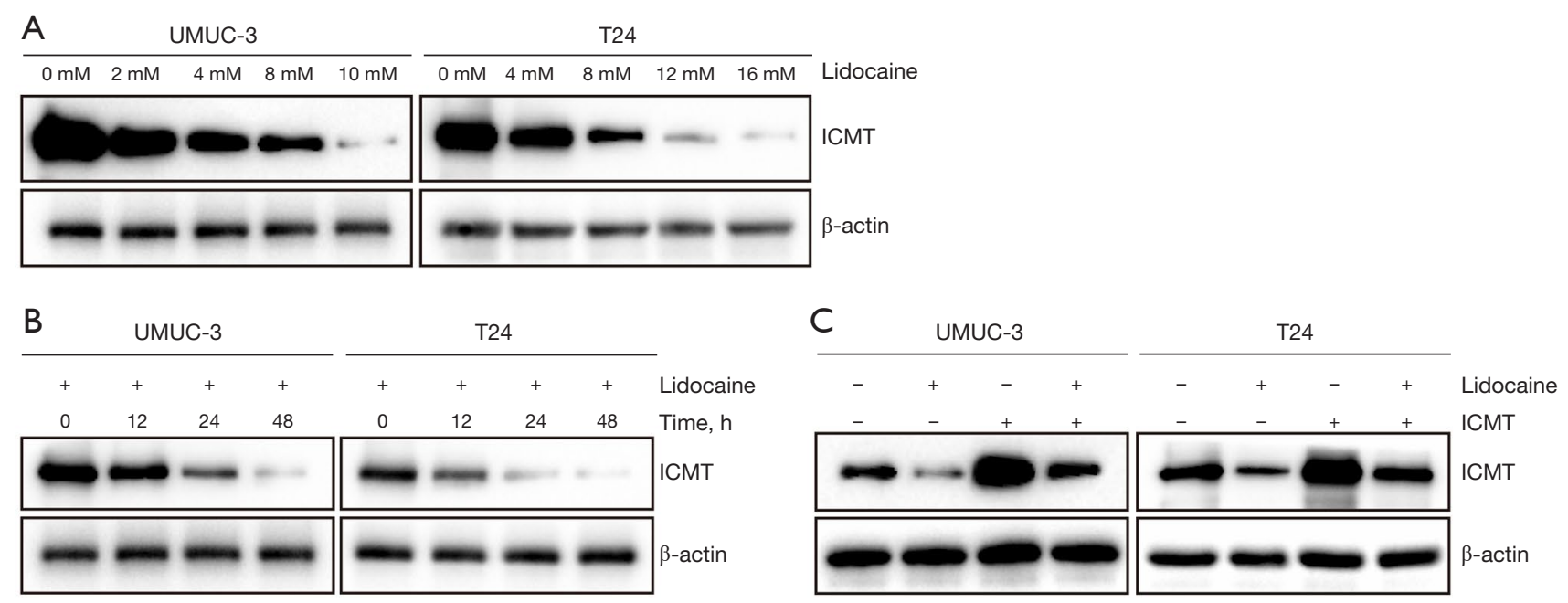

D

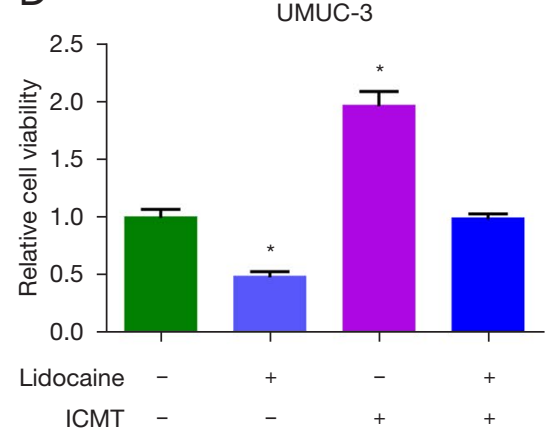

E

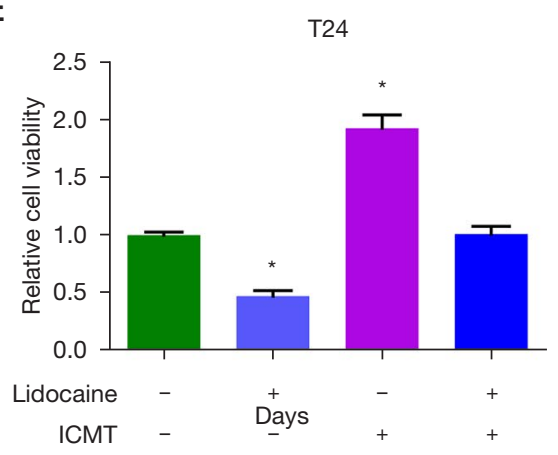

\section{$\mathrm{F}$}
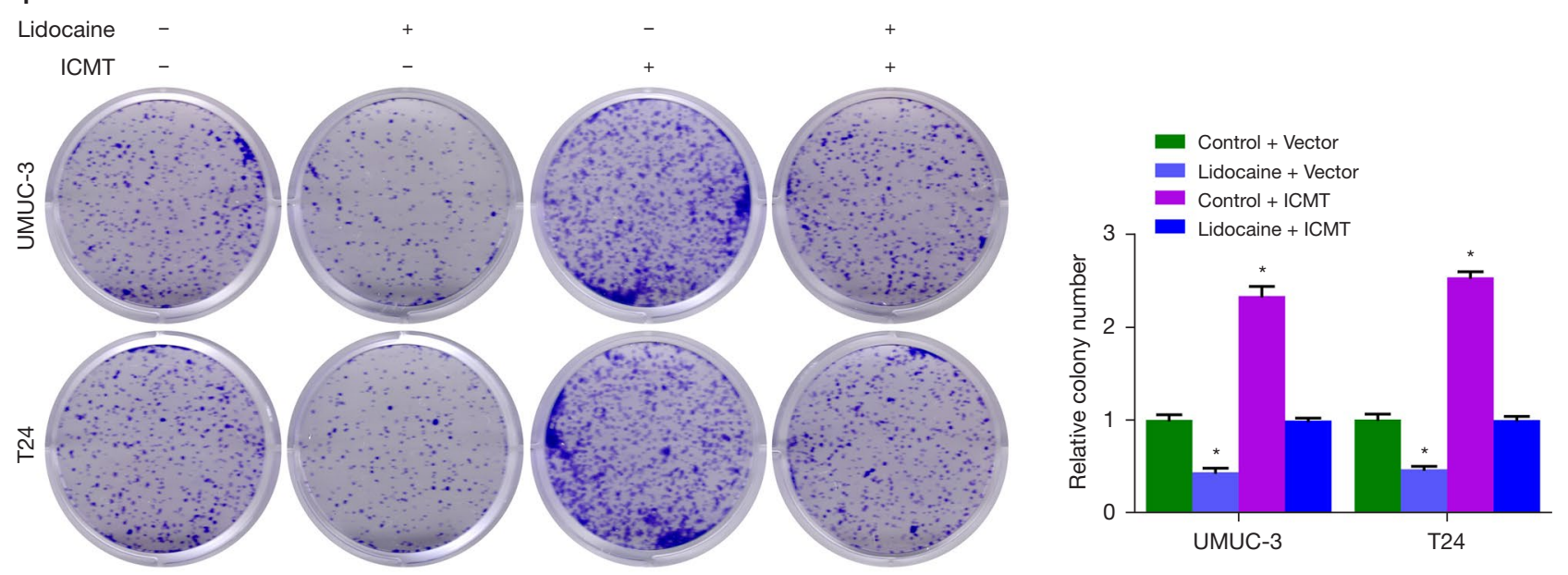

Figure 6 Lidocaine inhibits bladder cancer cell proliferation through ICMT. (A) After UMUC3 and T24 cells were treated with different concentrations of lidocaine, the protein level of ICMT was detected by western blotting; (B) after UMUC3 and T24 cells were treated with 4 and $8 \mathrm{mM}$ lidocaine for different times, ICMT protein levels were detected by western blotting. After the cells were treated with lidocaine and ICMT overexpression, respectively or simultaneously; (C) Western blot was used to detect the protein level of ICMT; (D,E) the survival of UMUC3 and T24 cells was measured using CCK-8 analysis; and (F) colony formation experiment (crystal violet staining) was used to determine the colony formation ability of UMUC3 and T24 cells. *, $\mathrm{P}<0.05$ vs. Control + Vector group. 
lidocaine and ICMT overexpression, respectively or simultaneously, and the ICMT protein was successfully overexpressed (Figure 6C). Then we further measured cell survival and colony formation. The results showed that ICMT overexpression reversed the inhibition of lidocaine on cell survival and colony formation (Figure $6 D-6 F$ ). In summary, these results confirmed that lidocaine inhibited cell proliferation by inhibiting ICMT expression.

\section{Discussion}

Bladder cancer is one of the most fatal malignant tumors in humans, with high incidence, high recurrence rate, and high mortality. Over the past 2 decades, local or metastatic bladder cancer has a poor prognosis and a low 5 -year survival rate due to uncontrolled proliferation, apoptosis, and metastasis of tumor cells (14). Studies have shown that local anesthetics may be beneficial to the treatment of cancer. Lidocaine is the most commonly used local anesthetic in clinic, which can inhibit the proliferation, invasion, and migration of tumor cells and induce apoptosis of tumor cells $(4,15)$. Recent studies have found that lidocaine can inhibit the proliferation of human bladder cancer cells, but there have been few studies on its specific downstream molecular mechanism (5). In this study, CCK-8 and cell colony analysis showed that the $\mathrm{IC}_{50}$ values $(48 \mathrm{~h})$ in UMUC3 and T24 cells were 3.92 and $7.84 \mathrm{mM}$, respectively. After $48 \mathrm{~h}$ treatment with 4 and $8 \mathrm{mM}$ lidocaine, the survival rate and colony formation ability of tumor cells decreased significantly in a dose- and time-dependent manner. The results in vivo showed that lidocaine significantly inhibited the tumor growth of tumor mice, confirming the anti-tumor effect of lidocaine in bladder cancer. In addition, LDH cytotoxicity test was used to evaluate the cytotoxicity of lidocaine in 2 cell lines. The results showed that lidocaine significantly increased LDH levels in UMUC3 and T24 cells. In order to clarify the mechanism of anti-proliferation effect of lidocaine on bladder cancer cells, we studied the effect of lidocaine treatment on cell cycle progression of UMUC3 and T24. The results of flow cytometry showed that lidocaine caused cell cycle arrest in G1. Therefore, it can be inferred that the inhibition of proliferation of bladder cancer cells by lidocaine is caused by cell cycle arrest. This is consistent with the results reported by Chang et al. (15) that lidocaine reduces the viability of thyroid cancer cells through G1 arrest. Western blot analysis showed that lidocaine treatment significantly down- regulated the expression of ki67, CDK2, and CCNB1 proteins, indicating that lidocaine-induced cell cycle arrest may be achieved by regulating cyclin family and CDK family proteins.

Network pharmacology and bioinformatics analysis of TCGA patient samples provide a new perspective for the detection and treatment of bladder cancer. Previous studies have shown that ICMT is involved in the growth, drug resistance, and progression of many cancers (16-18). In our study, we found that ICMT is one of many targets of lidocaine, and the expression of ICMT is up-regulated in TCGA-BLCA patients, suggesting that the abnormal expression of ICMT is related to tumorigenesis. We further found that ICMT was up-regulated in the late stage of bladder cancer and related to adverse pathological $\mathrm{T}$ staging. High expression of ICMT is associated with poor survival and high recurrence rate. In addition, the effect of ICMT expression on the proliferation of bladder cancer cells was verified by in vitro transfection of ICMT overexpression and knock-down plasmid. This study found that overexpression of the ICMT gene can significantly promote the proliferation of UMUC3 and T24 cells. On the contrary, knockdown of the ICMT gene inhibits cell proliferation, which is consistent with the results of $\mathrm{Xu}$ et al. (18). These results suggest that ICMT may be a potential biomarker for the diagnosis and treatment of bladder cancer.

The direct effects of lidocaine on cancer cells include inhibiting proliferation, inducing apoptosis, and inhibiting metastasis efficiency $(15,19,20)$. In particular, proliferation and apoptosis are 2 important signs of cancer cells, and inhibiting cell proliferation and inducing apoptosis are 2 promising strategies for anti-tumor development (21). The BCL2 family plays an important role in apoptosis, including anti-apoptotic proteins (BH1-4 domain) and pro-apoptotic proteins (BH1-3 and $\mathrm{BH} 3$ domain) (22). Specifically, the BCL2 subfamily is associated with inhibition of apoptosis, while the Bax subfamily promotes apoptosis (23). In this study, lidocaine treatment induced the apoptosis of UMUC3 and T24 cells. The mechanism may be through up-regulating the expression of pro-apoptotic protein Bax and down-regulating the expression of anti-apoptotic protein $\mathrm{Bcl}-2$, thereby activating the endogenous apoptotic pathway to trigger apoptosis. This is consistent with the results of lidocaine inducing HepG2 cell apoptosis by increasing the $\mathrm{Bax} / \mathrm{Bcl}-2$ ratio (4).

It is further speculated that lidocaine may inhibit the proliferation of bladder cancer cells by targeting ICMT. 
Our rescue experiments showed that the recovery of ICMT attenuated the inhibitory effect of lidocaine on the survival and growth of UMUC3 and T24 cells. The novelty of this study includes network pharmacology analysis of ICMT as a target gene of lidocaine and its clinical significance, application of 2 bladder cancer cell lines and rescue experiments. In summary, ICMT is a medium for lidocaine to inhibit the proliferation of bladder cancer cells. And We can screen and identify more key biomarkers of bladder cancer through TCGA and GEO data and a series of bioinformatics analysis.

\section{Conclusions}

Taken together, our results suggest that lidocaine affects the proliferation of bladder cancer cells by regulating cell cycle and Bax/Bcl-2 ratio. Lidocaine can down-regulate ICMT to inhibit the proliferation of bladder cancer cells.

\section{Acknowledgments}

Funding: None.

\section{Footnote}

Reporting Checklist: The authors have completed the ARRIVE reporting checklist. Available at https://dx.doi. org/10.21037/tau-21-893

Data Sharing Statement: Available at https://dx.doi. org/10.21037/tau-21-893

Conflicts of Interest: All authors have completed the ICMJE uniform disclosure form (available at https://dx.doi. org/10.21037/tau-21-893). The authors have no conflicts of interest to declare.

Ethical Statement: The authors are accountable for all aspects of the work in ensuring that questions related to the accuracy or integrity of any part of the work are appropriately investigated and resolved. The study was conducted in accordance with the Declaration of Helsinki (as revised in 2013). Experiments were performed under a project license (No. 2020067) granted by ethics committee of Harbin Medical University Cancer Hospital, in compliance with Animal Management Rule of China (Ministry of Health, China, document No. 552001).
Open Access Statement: This is an Open Access article distributed in accordance with the Creative Commons Attribution-NonCommercial-NoDerivs 4.0 International License (CC BY-NC-ND 4.0), which permits the noncommercial replication and distribution of the article with the strict proviso that no changes or edits are made and the original work is properly cited (including links to both the formal publication through the relevant DOI and the license). See: https://creativecommons.org/licenses/by-nc-nd/4.0/.

\section{References}

1. Fernández MI, Brausi M, Clark PE, et al. Epidemiology, prevention, screening, diagnosis, and evaluation: update of the ICUD-SIU joint consultation on bladder cancer. World J Urol 2019;37:3-13.

2. Seah DSE, Herschtal A, Tran H, et al. Subcutaneous Lidocaine Infusion for Pain in Patients with Cancer. J Palliat Med 2017;20:667-71.

3. Liu H, Wang Y, Chen B, et al. Effects of LidocaineMediated CPEB3 Upregulation in Human Hepatocellular Carcinoma Cell Proliferation In Vitro. Biomed Res Int 2018;2018:8403157.

4. Xing W, Chen DT, Pan JH, et al. Lidocaine Induces Apoptosis and Suppresses Tumor Growth in Human Hepatocellular Carcinoma Cells In Vitro and in a Xenograft Model In Vivo. Anesthesiology 2017;126:868-81.

5. Yang X, Zhao L, Li M, et al. Lidocaine enhances the effects of chemotherapeutic drugs against bladder cancer. Sci Rep 2018;8:598.

6. Sun X, Yan H. MicroRNA-99a-5p suppresses cell proliferation, migration, and invasion by targeting isoprenylcysteine carboxylmethyltransferase in oral squamous cell carcinoma. J Int Med Res 2021;49:300060520939031.

7. Liu Q, Chen J, Fu B, et al. Isoprenylcysteine carboxylmethyltransferase regulates ovarian cancer cell response to chemotherapy and Ras activation. Biochem Biophys Res Commun 2018;501:556-62.

8. Do MT, Chai TF, Casey PJ, et al. Isoprenylcysteine carboxylmethyltransferase function is essential for RAB4Amediated integrin $\beta 3$ recycling, cell migration and cancer metastasis. Oncogene 2017;36:5757-67.

9. Manu KA, Chai TF, Teh JT, et al. Inhibition of Isoprenylcysteine Carboxylmethyltransferase Induces Cell-Cycle Arrest and Apoptosis through p21 and p21Regulated BNIP3 Induction in Pancreatic Cancer. Mol 
Cancer Ther 2017;16:914-23.

10. Sun WT, Xiang W, Ng BL, et al. Inhibition of isoprenylcysteine carboxylmethyltransferase augments BCR-ABL1 tyrosine kinase inhibition-induced apoptosis in chronic myeloid leukemia. Exp Hematol 2016;44:18993.e2.

11. Pan Q, Liu R, Banu H, et al. Inhibition of isoprenylcysteine carboxylmethyltransferase sensitizes common chemotherapies in cervical cancer via Ras-dependent pathway. Biomed Pharmacother 2018;99:169-75.

12. Chamaraux-Tran TN, Mathelin C, Aprahamian M, et al. Antitumor Effects of Lidocaine on Human Breast Cancer Cells: An In Vitro and In Vivo Experimental Trial. Anticancer Res 2018;38:95-105.

13. Zhou J, Xu D, Xie H, et al. miR-33a functions as a tumor suppressor in melanoma by targeting HIF-1 $\alpha$. Cancer Biol Ther 2015;16:846-55.

14. Black AJ, Black PC. Variant histology in bladder cancer: diagnostic and clinical implica-tions. Transl Cancer Res 2020;9:6565-75.

15. Chang YC, Hsu YC, Liu CL, et al. Local anesthetics induce apoptosis in human thyroid cancer cells through the mitogen-activated protein kinase pathway. PLoS One 2014;9:e89563.

16. Zhou N, Chi ZP, Li WJ, et al. Effects of isoprenylcysteine carboxylmethyltransferase silencing on the migration and invasion of tongue squamous cell carcinoma. Hua Xi Kou
Qiang Yi Xue Za Zhi 2021;39:328-35.

17. Borini Etichetti C, Di Benedetto C, Rossi C, et al. Isoprenylcysteine carboxy methyltransferase (ICMT) is associated with tumor aggressiveness and its expression is controlled by the p53 tumor suppressor. J Biol Chem 2019;294:5060-73.

18. Xu J, Zhu Y, Wang F, et al. ICMT contributes to hepatocellular carcinoma growth, survival, migration and chemoresistance via multiple oncogenic pathways. Biochem Biophys Res Commun 2019;518:584-9.

19. Piegeler T, Schläpfer M, Dull RO, et al. Clinically relevant concentrations of lidocaine and ropivacaine inhibit TNF $\alpha$ induced invasion of lung adenocarcinoma cells in vitro by blocking the activation of Akt and focal adhesion kinase. Br J Anaesth 2015;115:784-91.

20. Chang YC, Liu CL, Chen MJ, et al. Local anesthetics induce apoptosis in human breast tumor cells. Anesth Analg 2014;118:116-24.

21. Hanahan D, Weinberg RA. Hallmarks of cancer: the next generation. Cell 2011;144:646-74.

22. Inoue-Yamauchi A, Jeng PS, Kim K, et al. Targeting the differential addiction to anti-apoptotic BCL-2 family for cancer therapy. Nat Commun 2017;8:16078.

23. Birkinshaw RW, Czabotar PE. The BCL-2 family of proteins and mitochondrial outer membrane permeabilisation. Semin Cell Dev Biol 2017;72:152-62.
Cite this article as: Teng X, Liu Y, Wang L, Wang G. Lidocaine exerts anticancer activity in bladder cancer by targeting isoprenylcysteine carboxylmethyltransferase (ICMT). Transl Androl Urol 2021;10(11):4219-4230. doi: 10.21037/ tau-21-893 